\title{
Salivary Cyanide and Thiocyanate Levels in Smokers with Periodontitis
}

\author{
${ }^{1}$ Ghaida Okasha, ${ }^{2}$ Ibrahim Haddad, ${ }^{3}$ Ali A Sulaiman
}

\begin{abstract}
Aim: Several recent studies have indicated that a number of salivary biomarkers seem to be compromised in smokers with periodontal disease. The aim of this study is to assess salivary levels of cyanide and thiocyanate in Syrian patients with chronic $(\mathrm{ChP})$ and aggressive (AgP) periodontitis, and to investigate the effect of smoking on these two biomarkers.
\end{abstract}

Material and methods: The study population comprised 162 individuals allocated within four groups: chronic periodontitis $(\mathrm{ChP})$, elderly control group (EC), aggressive periodontitis $(\mathrm{AgP})$, Young control group (YC). All participants were asked to follow certain rules for at least 2 hours before the collection of morning samples of unstimulated whole saliva. Levels of Cyanide $(\mathrm{CN})$ and thiocyanate (SCN) were estimated using a spectrophotometer. Statistical analysis was performed to examine differences between study groups.

Results: Salivary CN and SCN levels were significantly higher in $\mathrm{AgP}$ and $\mathrm{ChP}$ groups compared to healthy subjects. Smokers in disease groups had a significant increase in $\mathrm{CN}$ and SCN compared to nonsmokers.

Conclusion: This study exhibited significantly elevated levels of salivary $\mathrm{CN}$ and $\mathrm{SCN}$ in smokers with periodontitis as opposed to nonsmokers.

Clinical significance: Salivary $\mathrm{CN}$ and SCN could be considered as important indicators for monitoring periodontitis.

Keywords: Cyanide, Clinical laboratory research, Periodontitis, Saliva, Smoking, Thiocyanate.

How to cite this article: Okasha G, Haddad I, Sulaiman AA. Salivary Cyanide and Thiocyanate Levels in Smokers with Periodontitis. World J Dent 2018;9(6):462-467.

Source of support: Nil

Conflict of interest: None

\section{INTRODUCTION}

Saliva is an important biological fluid with clinically informative properties that can provide novel approaches in diagnosis, monitoring, and management of patients

\footnotetext{
1,3 Department of Periodontology, Faculty of Dentistry, Damascus University, Damascus, Syria

${ }^{2}$ Department of Life Science, Faculty of Dentistry, Damascus University, Damascus, Syria
}

Corresponding Author: Ali A Sulaiman, Department of Periodontology, Faculty of Dentistry, Damascus University, Damascus, Syria, Phone: 00963-956790836, e-mail: dr.alisulaiman43@gmail. com with both oral and systemic diseases. It is easily collected and stored, and contains specific soluble biological markers that make it ideal for early detection of certain diseases. ${ }^{1}$

Periodontal diseases are chronic inflammatory entities that are initiated by bacterial plaque deposited on the adjacent teeth and characterized by infiltration of leukocytes, loss of connective tissues and alveolar bone resorption. Periodontitis is a complex multifactorial disease that is affected by the interaction between bacteria, multiple genes, and environmental factors. Smoking is one of the most important risk factors for periodontitis. The effects of smoking manifest as greater attachment loss, deeper periodontal pockets and a significant increase in bone loss. Hence, periodontitis is more prevalent and severe among smokers. ${ }^{2}$

Historically, clinical and radiographic measures of periodontal tissue destruction have been the main go-to means for diagnosis of periodontal disease. However, over the past few years, there has been increasing interest in the use of saliva for the diagnosis of oral and systemic diseases. ${ }^{1}$ The tendency to use human saliva for diagnostic purposes has gained momentum due to its noninvasive nature and decreased infection risk as opposed to chemical analysis of blood and/or urine samples. ${ }^{3}$

Numerous studies have investigated the composition, flow rate, $\mathrm{PH}$, buffer capacity, ions, proteins and a number of other components in the salivary samples from patients with varying degrees of periodontitis. Consequently, based upon the finding of these studies certain salivary properties and constituents were suggested as possible biomarkers for diagnosing and monitoring periodontal diseases.

Cyanide is a highly toxic chemical and an essential reagent for many industrial processes. It occurs naturally in plant seeds as a cyanogenic glycoside. Furthermore, cyanide may also be present in drinking water, soil, and air. Human exposure to cyanide is also associated with tobacco smoking. ${ }^{4}$

Prolonged exposure to cyanide leads to the weakening of the body, the occurrence of many diseases and even death. ${ }^{5}$

Salivary thiocyanate $(\mathrm{SCN})$, a metabolic product of cyanide, is a normal component of numerous body fluids such as saliva, tears, and serum. Its main sources in the 
human body are diet and tobacco. SCN is secreted in saliva and has a long half-life of 10-14 days in normal adults. ${ }^{6}$

Normal nonsmokers have salivary SCN concentration in ranges from 0.5 to $2 \mathrm{~mm}$. However, heavy smokers may have a salivary concentration that can reach up to $6 \mathrm{~mm}$. SCN has been shown to be a chemical indicator in smokers and smokeless tobacco users. Measurement of $\mathrm{CN}$ and SCN are of growing importance in monitoring some clinical conditions and distinguishing tobacco smokers from nonsmokers and determining some clinical conditions. ${ }^{7}$ Aggarwal et al. indicated a significant increase in SCN levels in saliva of cigarette smokers as compared to nonsmokers and suggested that salivary SCN can be used as a safe and acceptable prevalence measurement for cigarette smoking behavior. ${ }^{8}$

A review of the literature revealed limited studies examining $\mathrm{CN}$ levels in saliva of patients with periodontitis. On the other hand, there are a handful of studies collating and associating the salivary levels of SCN in smokers with chronic periodontitis to other patient groups. ${ }^{6}$

The aim of this study was to examine the salivary levels of cyanide and thiocyanate in patients with periodontitis (chronic and aggressive) and to investigate any possible association between smoking and these two salivary biomarkers.

\section{MATERIALS AND METHODS}

\section{Study Population}

The study was approved by the Institutional Ethics Committee at Damascus University.

Patients referred to the Department of Periodontology, Faculty of Dentistry, Damascus University were invited to participate in the study. In total, 162 subjects were enrolled in the study. Thorough medical and dental histories were obtained and a complete periodontal chart was recorded for each participant. Examined periodontal parameters included: plaque index (PI), gingival index (GI), and probing pocket depth (PPD). Panoramic dental tomographs were used to confirm the diagnosis.

Subjects were excluded if they met any of these conditions: had less than 20 teeth (other than third molars), had any systemic disease, underwent treatment with antibiotics over the past three months, pregnancy, breastfeeding and had any diseases or conditions that affect the secretion of saliva.

Finally, all participants gave their written consent after being informed about the nature of the study and its purpose.

Participants were categorized into four groups according to the guidelines of the American Academy of Periodontology 1999:
- Chronic periodontitis group (ChP): Comprised 44 patients (13 females and 31 males, aged $\geq 45$ years; mean age 51 years) and have 2 interproximal sites with $C A L \geq 3 \mathrm{~mm}$ and 2 or more interproximal sites with PPD $\geq 4 \mathrm{~mm}$ (not on the same tooth).

- Elderly control group (EC): Consisted of 40 age-sex matched subjects ( 18 female and 22 males, aged $\geq 45$ years; mean age 46 years) had no periodontal disease and were matching to $\mathrm{ChP}$ group in race and age.

- Aggressive periodontitis group (AgP): Comprised 32 patients (17 females and 15 males, aged <35 years; mean age 28 years) with PPD $>4 \mathrm{~mm}$ around at least three teeth other than the first molars and incisors.

- Young control group (YC): Consisted of 46 age-sex matched subjects (20 females and 26 males, aged $<35$ years; mean age 27 years) and had healthy periodontium and were matching to group AgP.

Subjects in all study groups were categorized as smokers if they had $>5$ cigarettes per day for the past 3 years.

\section{Collection of Samples}

Subjects were asked to refrain from drinking (except water), food intake, chewing gum and tooth brushing within 2 hours of sampling. All saliva samples were collected at appointments between 11 am and 12 noon. Whole unstimulated saliva was collected by spitting without swallowing with the subject seated in an upright position. Sterilized plastic tubes containing the collected saliva were stored at $-20^{\circ}$ until future use.

\section{Laboratory Studies}

\section{Cyanide Determination}

Spectrophotometric determination of cyanide was performed according to the method described by Pitschmann et al. ${ }^{9}$ In brief, a tube was filled with measured $2 \mathrm{~mL}$ of a standard solution with added $0.2 \mathrm{~mL}$ of sodium hydroxide solution, $2 \mathrm{~mL}$ of $\mathrm{p}$-nitrobenzaldehyde solution, $2 \mathrm{~mL}$ of tetrazolium blue solution, and completed by $10 \mathrm{~mL}$ of methanol. After 30 minutes, the formed red-purple color was measured at a wavelength of $520 \mathrm{~nm}$ as opposed to methanol.

The values for creating a calibration graph were acquired by measuring a series of standard working solutions of cyanides in different concentrations. Values measured in blind determinations with distilled water were subtracted from values measured in the individual standards. Statistical evaluation of the method was carried out in the usual way; the concentration of cyanides in the formulas was determined directly from the calibration graph, with regard to the dilution or by the Lambert-Beer law. 


\section{Thiocyanate Determination}

The procedures to determine thiocyanate levels were conducted according to the method described by Lahti et al. ${ }^{10}$ Briefly, after centrifugation, a $0.5 \mathrm{~mL}$ of supernatant saliva was mixed with $9.5 \mathrm{~mL} \mathrm{Fe}\left(\mathrm{NO}_{3}\right)_{3}$ reagent. Subsequently, the following reaction occurs: $\mathrm{Fe}^{+3}(\mathrm{aq})+\mathrm{SCN}(\mathrm{aq}) \rightarrow$ $\mathrm{FeSCN}^{2+}$ (aq). The resulting $\mathrm{FeSCN}^{2+}$ complex exhibit deep red color, which can be conveniently measured with UV spectrophotometry at $447 \mathrm{~nm}$ wave lengths. The concentration of thiocyanate in the saliva solution was calculated through Lambert-Beer law.

\section{Statistical Analysis}

Statistical testing was performed using. SPSS (version 21, Chicago, IL, USA). The Chi-square test was used to analyze the differences in age and sex between study groups.

The Mann-Whitney U and one-way ANOVA tests were used to analyze levels of $\mathrm{CN}$ and SCN among study groups. Finally, intergroup comparisons were carried out by using the Bonferroni multiple comparison tests.

Spearman rank correlation test was used to assess the relationship between the clinical indices and salivary $\mathrm{CN}$ and SCN. Statistical significance was aetat values of $p<0.05$.

\section{RESULTS}

Table 1 shows the main characteristics of the study population. In this study, a total of 162 subjects were recruited. There were 21 (47.7\%) smokers and $23(52.3 \%)$ nonsmokers in ChP group. Whilst group EC had 16 (40\%) smokers and
$24(60 \%)$ nonsmokers. Likewise, There were 13 (47.7\%) smokers and 23 (52.3) nonsmokers in AgP group compared to $24(52.2 \%)$ smokers and $22(47.8 \%)$ nonsmokers in YC group (Table 1).

The mean GI, PI, and BOP are presented in Table 2. In general, higher measurement values of the examined clinical parameters were recorded in both disease groups as opposed to the corresponding control groups, especially among smokers (Table 2).

The mean salivary levels of $\mathrm{CN}$ and $\mathrm{SCN}$ are presented in Table 3. The mean CN level from patients with $\mathrm{ChP}$ was $1.59 \mathrm{mg} / \mathrm{L}$ compared to $1.018 \mathrm{mg} / \mathrm{L}$ in EC group. This difference was highly significant $(p<0.001)$. Similarly, there was a significant difference $(p=0.03)$ in the mean level of $\mathrm{CN}$ in patients with $\mathrm{AgP}$ compared to subjects in YC group (Table 3).

Similarly, the mean level of SCN was $141.5 \mathrm{mg} / \mathrm{L}$ and $90.52 \mathrm{mg} / \mathrm{L}$ in ChP and EC groups respectively. This difference was highly significant $(p<0.001)$. Likewise, SCN was significantly higher in $\mathrm{AgP}$ group $(102.4 \mathrm{mg} / \mathrm{L})$ as opposed to the corresponding YC controls $(85.5 \mathrm{mg} / \mathrm{L})$ (Table 3).

As can be seen in Table 4, the Spearman correlation test revealed highly significant correlation $(p<0.001)$ between salivary levels of $\mathrm{CN}$ and SCN and both PI and GI indices among patients with AgP. However, there was no significant correlation between $\mathrm{CN}$ and $\mathrm{SCN}$ salivary levels and PI, GI and BOP indices in ChP group (Table 4).

The data from Table 5 show that smoking patients from $\mathrm{AgP}$ and $\mathrm{ChP}$ groups had a highly significant $(p<0.001)$ salivary levels of $\mathrm{CN}$ and $\mathrm{SCN}$ as opposed to the corresponding control groups and nonsmokers (Table 5).

Table 1: Parameters of the study groups

\begin{tabular}{|c|c|c|c|c|c|}
\hline \multicolumn{6}{|c|}{ Groups of study } \\
\hline$N$ & & $Y C$ & $E C$ & $A g P$ & $\mathrm{ChP}$ \\
\hline Age mean (years) & & 46 & 40 & 32 & 44 \\
\hline \multirow[t]{2}{*}{$\operatorname{Sex} N(\%)$} & Female & \pm 276 & $6 \pm 46$ & $28 \pm 6$ & \pm 519 \\
\hline & Male & 20 & 18 & 17 & 13 \\
\hline \multirow[t]{7}{*}{ Smoking N (\%) } & Nonsmoking & $(43.5 \%)$ & $(45 \%)$ & $(53.1 \%)$ & $(29.5 \%)$ \\
\hline & Smoking & 26 & 22 & 15 & 31 \\
\hline & & $(56.5 \%)$ & $(55 \%)$ & $(46.9 \%)$ & $(70.5 \%)$ \\
\hline & & 22 & 24 & 19 & 23 \\
\hline & & $(47.8 \%)$ & $(60 \%)$ & $(59.4 \%)$ & $(52.3 \%)$ \\
\hline & & 24 & 16 & 13 & 21 \\
\hline & & $(52.2 \%)$ & $(40 \%)$ & $(40.6 \%)$ & $(47.7 \%)$ \\
\hline
\end{tabular}

$\mathrm{YC}=$ Young Control group, $\mathrm{EC}=$ Elderly Control group, $\mathrm{AgP}=$ Aggressive periodontitis group, $\mathrm{ChP}=$ Chronic Periodontitis group

Table 2: Mean clinical periodontal indices in study groups

\begin{tabular}{lllll}
\hline & $Y C$ & $E C$ & $A G P$ & $C H P$ \\
\hline $\mathrm{GI}$ & $1.08 \pm 0.47$ & $1.36 \pm 0.49$ & $1.45 \pm 0.45$ & $1.37 \pm 0,49$ \\
$\mathrm{PI}$ & $1.18 \pm 0.56$ & $1.39 \pm 0.56$ & $1.4 \pm 0.59$ & $2.3 \pm 0,56$ \\
$\mathrm{BOP}$ & $19.89 \pm 24.89$ & $23.84 \pm 26.26$ & $36.37 \pm 29.56$ & $38.61 \pm 30.22$ \\
\hline
\end{tabular}

$\mathrm{GI}=$ Gingival index, $\mathrm{PI}=$ Plaque index, $\mathrm{BOP}=$ Bleeding on probing 
Salivary Cyanide and Thiocyanate Levels in Smokers with Periodontitis

Table 3: differences in mean salivary $\mathrm{CN}$ and SCN levels among study groups

\begin{tabular}{|c|c|c|c|c|c|c|c|c|c|c|}
\hline & \multirow[b]{2}{*}{ ChP } & \multirow[b]{2}{*}{$E C$} & \multicolumn{2}{|c|}{ ChP vs EC } & \multirow[t]{2}{*}{$A g P$} & \multirow[t]{2}{*}{$Y C$} & \multicolumn{2}{|c|}{$A g P$ vs $Y C$} & \multicolumn{2}{|c|}{ ChP vs $A g P$} \\
\hline & & & $Z^{*}$ & $p$ & & & $Z^{*}$ & $p$ & $Z^{*}$ & $p$ \\
\hline $\mathrm{CN}$ & $1.59 \pm 0,85$ & $1.018 \pm 0.76$ & -3.97 & 0.001 & $1.2 \pm 0.84$ & $0.97 \pm 0.84$ & 2.14 & 0.03 & -2.34 & 0.019 \\
\hline SCN & $141.5 \pm 70.8$ & $90.52 \pm 69.1$ & -4.07 & 0.001 & $102.4 \pm 80.2$ & $85.5 \pm 75.6$ & $2.17-$ & 0.03 & $2.91-$ & 0.004 \\
\hline
\end{tabular}

*Mann-Whitney test

Table 4: Correlation between salivary levels of $\mathrm{CN}$ and SCN and clinical parameters in the study groups

\begin{tabular}{llllll}
\hline \multirow{2}{*}{ Study groups } & Clinical parameters & \multicolumn{2}{c}{ CN } & \multicolumn{2}{c}{ SCN } \\
\cline { 3 - 6 } AgP & PI & $0.040-$ & 0.722 & $0.048-$ & $0.022-$ \\
& GI & $0.034-$ & 0.759 & 0.022 & 0.844 \\
& BOP & $0.100-$ & 3.72 & $0.082-$ & 0.462 \\
\hline ChP & PI & $0.086-$ & 0.455 & $0.101-$ & 0.378 \\
& GI & $0.397-$ & 0.000 & $0.395-$ & 0.000 \\
& BOP & $0.335-$ & 0.003 & $0.324-$ & 0.000 \\
\hline
\end{tabular}

*Spearman correlation coefficient

Table 5: Multiple comparisons of salivary $\mathrm{CN}$ and SCN among disease groups

\begin{tabular}{llll}
\hline & & & $p^{*}$ \\
\hline \multirow{4}{*}{ CN } & AgP S & AgP NS & 0,000 \\
& & ChP S & 0,247 \\
& & ChP NS & 0,000 \\
& ChP S & AgP S & 0,000 \\
& & AgP NS & 0,000 \\
& & ChP NS & 0,130 \\
\hline \multirow{4}{*}{ SCN } & AgP S & AgP NS & 0,000 \\
& & ChP S & 0,241 \\
& ChP S & ChP NS & 0,000 \\
& & AgP S & 0,241 \\
& & AgP NS & 0,000 \\
& & ChP NS & 0,018 \\
\hline
\end{tabular}

AgP S = Aggressive prriodontitis smoking, AgP NS = Aggressive prriodontitis non-smoking, ChP S = Chronic periodontitis smoking, ChP NS = Chronic periodontitis non-smoking *Bonferroni test

\section{DISCUSSION}

Over the past decade, saliva has gained increasing interest as compared to other biological fluids such as blood and urine. Assessment of saliva composition and properties has been used to better understand variations in oral diseases. In this regard examination of saliva has become a contributory aid in the assessment of risk factors and the evaluation of disease progression and would constitute an important tool in the clinical diagnosis of active periodontitis.

To the best of our knowledge, this study is the first report that attempted to analyze salivary $\mathrm{CN}$ levels in patients with $\mathrm{ChP}$ and AgP. Moreover, the present study is the first report to assess salivary SCN levels among patients with $\mathrm{AgP}$ as well as comparing its correlation with smoking.

The present study showed significantly higher levels ( $\mathrm{p}<001)$ of salivary $\mathrm{CN}$ and SCN in patients with ChP and AgP compared to periodontally healthy subjects. Furthermore, $\mathrm{CN}$ and SCN levels were significantly higher $(p<0.05)$ in smokers when compared to nonsmokers among patients in both $\mathrm{ChP}$ and $\mathrm{AgP}$ groups. These findings were in line with results of previous studies. Data obtained from the study of Kalburgi et al. exhibited a significant increase in salivary SCN among smokers and gutka chewers with $\mathrm{ChP}$ as compared to healthy and nonsmokers with ChP. ${ }^{11}$ Similarly, Hedge et al. reported a significant increase in SCN salivary levels in smokers with $\mathrm{ChP}$ as compared to nonsmokers. ${ }^{12}$

The notoriety of cyanide has been known to mankind since antiquity. Cyanide is a mitochondrial toxin that impairs cellular respiration, causing morbidity or mortality within a short time. Cyanide's actions are complex and cannot be attributed solely to the deprivation of cellular oxygen. Recent mechanistic studies show that cyanide inhibits multiple enzymes and alters several vital intracellular processes that lead to a cascade of toxic events. ${ }^{13}$

$\mathrm{CN}$ has fast absorption and distribution into tissues and the bloodstream, binding to metalloenzymes and rendering them inactive. A small amount of cyanide intake occurs during the process of smoking tobacco. Consequently, cyanide and thiocyanate concentrations in the plasma, whole blood, saliva, and tissues of such smokers are elevated relative to non-smokers. in certain settings, chronic cyanide exposure through inhalation of tobacco cigarette smoke has been associated with the disease. ${ }^{14}$

Cyanide has been previously shown to induce oxidative stress and damage in a number of biological systems. Cyanide-induced oxidative stress may be due to increases in reactive oxygen species (ROS) and nitric oxide as well as to inhibition of antioxidant system and mitochondrial function. There are numerous evidence that oxidative stress has been implicated in diverse pathological conditions. ${ }^{15}$ 
It has been revealed that $\mathrm{ChP}$ has been associated with lower levels of total antioxidant capacity and oxidative stress has been considered a strong feature in periodontitis. Also, salivary peroxidase plays dual role acting as both an antibacterial and as antioxidant vector. This enzyme is of great importance in the oral defense mechanism especially against the attack of ROS. However, in smokers, it has a decreased activity due to the cyanide ions present in cigarette smoke. ${ }^{16}$

On another note, this study demonstrated a negative correlation between GI and BOP parameters in smokers with $\mathrm{AgP}$ and $\mathrm{ChP}$ and salivary levels of $\mathrm{CN}$ and SCN. This finding is in agreement with the findings of a number of recent studies which have reported that salivary SCN levels are in an inverse relationship with gingival inflammation and plaque. ${ }^{11}$

The result of our study can be attributed to the detrimental effects of smoking on humans. Smoking has been identified as a major environmental risk factor associated with increased incidence and severity of periodontitis. ${ }^{17}$ Tobacco use leads to a change in the production or composition of saliva along with an alteration in the mucosal permeability and can predispose to oral cancer. Additionally, there is higher periodontal pocket depth and loss of attachment in smokers. Nevertheless, it is noticed that nicotine causes vasoconstriction of gingival blood vessels leading to less bleeding on probing in smokers. Hence, the compromised salivary system makes the periodontium more susceptible to the disease process and the clinical symptoms continue to occur at a slower rate. ${ }^{18}$

Ideally, the immune system should be capable of reducing the harmful bacteria in the mouth that cause periodontal disease. However, the chemicals in tobacco, including carbon monoxide, nicotine, tar, and cyanide not only increase the accumulation of plaque and calculus that can irritate the gingiva and cause infection but also affect the immune system, reducing the ability to fight off these pathogens and heal the damaged periodontium properly. ${ }^{19}$

\section{Limitations of the Study}

It is noteworthy to mention that the main shortcoming of this study was related to making a direct comparison to previous work on the investigated subject. This task was not an as smooth process as one might expect due to the lack of similar studies in the literature especially the ones examining the levels of $\mathrm{CN}$ in the saliva of patients with $\mathrm{ChP}$ and AgP.

\section{CONCLUSION}

Based upon the results obtained of this study, it can be concluded that patients who were smokers with chronic and aggressive periodontitis have significantly increased levels of salivary cyanide and thiocyanate as compared to nonsmokers. Thus, it is suggested that salivary $\mathrm{CN}$ and $\mathrm{SCN}$ can be considered a useful reliable biomarker that can help in following patients with periodontitis.

\section{REFERENCES}

1. Malamud D. Saliva as a diagnostic fluid. Dental Clinics. 2011;55(1):159-178.

2. Lindhe J. Clinical periodontology and implant dentistry. Oxford: Wiley-Blackwell; 2015.

3. Madalli VB, Basavaraddi SM, Burde K, Horatti P. Saliva-A diagnostic tool. JDMS. 2013;11(6):96-99.

4. Jaszczak E, Narkowicz S, Namiesnik J, Polkowska Z. Determination of cyanide in urine and saliva samples by ion chromatography with pulsed amperometric detection. Monatshefte fur chemie. 2017;148(9):1645-1649.

5. Abbasi S, Valinezhad R, Khani H. A novel kinetic spectrophotometric method for the determination of ultra-trace amount of cyanide. Spectrochim Acta A. 2010;77:112-116.

6. Kalburgi CV, Naik KL, Kokatnur MV, Warad S. Estimation and correlation of salivary thiocyanate levels in healthy and different forms of tobacco users having chronic periodontitis: A cross-sectional biochemical study. Contemporary clinical dentistry. 2014;5(2):182-186.

7. Paul BD, Smith ML. Cyanide and thiocyanate in human saliva by gas chromatography-mass spectrometry. Journal of analytical toxicology. 2006;30(8):511-515.

8. Aggarwal A, Keluskar V, Goyal R, Dahiya P. Salivary thiocyanate: a biochemical indicator of cigarette smoking in adolescents. Oral health \& preventive dentistry. 2013;11(3).

9. Pitschmann V KZ, Tušarová I. A simple spectrophotometric determination of cyanides by pnitrobenzaldehyde and tetrazolium blue. Advances in Military Technology. 2011;6(2).

10. Hovinen J, Lahti M, Vilpo J. Spectrophotometric determination of thiocyanate in human saliva. Journal of chemical education. 1999;76(9):1281.

11. Kalburgi CV, Naik KL, Kokatnur MV, Warad S. Estimation and correlation of salivary thiocyanate levels in healthy and different forms of tobacco users having chronic periodontitis: A cross-sectional biochemical study. Contemporary clinical dentistry. 2014;5(2):182.

12. Hegde S, Chatterjee E, Rajesh K, Kumar MA. Estimation and correlation of salivary thiocyanate levels in periodontally healthy subjects, smokers, nonsmokers, and gutka-chewers with chronic periodontitis. Indian journal of dental research. 2016;27(1):12.

13. Jing Liu CU, Linnzi Wright, Carey Pope. Handbook of Toxicology of Chemical Warfare Agents (Second Edition). second ed: Elsevier; 2015. p. 301-314.

14. Hall AH, Isom GE, Rockwood GA, editors. Toxicology of Cyanides and Cyanogens: Experimental, Applied and Clinical Aspects. John Wiley \& Sons; 2015 Dec 14..

15. Ghodsi V, Baghshani H. Effects of prolonged oral exposure to cyanide on some oxidative stress biomarkers in blood of rats. Romanian Journal of Biochemistry. 2013;50(2): 101-108.

16. Ekuni D. Studies on periodontal disease. [Place of publication not identified]: Humana; 2016. 
17. Cope G, Nayyar P, Holder R, Brock G, Chapple I. Near-patient test for nicotine and its metabolites in saliva to assess smoking habit. Annals of clinical biochemistry. 2000;37(5):666-673.

18. Peji A, Obradovi R, Kesi L, Kojovi D. Smoking and periodontal disease a review. Med Biol. 2007;14(2):53-59.
19. Barbour SE, Nakashima K, Zhang J-B, Tangada S, Hahn C-L, Schenkein HA, et al. Tobacco and smoking: environmental factors that modify the host response (immune system) and have an impact on periodontal health. Critical Reviews in Oral Biology \& Medicine. 1997;8(4):437-460. 